\title{
The polymerase chain reaction patents: going, going, ... still going
}

\author{
David McDowell
}

J R Soc Med 2006;99:62-64

\section{INTRODUCTION}

The story of the polymerase chain reaction $(\mathrm{PCR})$ rumbles on. The foundational patents for this key technology expired in March last year in the USA and will do the same in Europe in March 2006. Will this result in a genetic freefor-all as many have predicted with the demise of licence and royalty fees? The answer is far from clear.

So what is polymerase chain reaction and why all the hype. Genetics affects all living things. It is what makes us different, not just at a superficial physical level, but in ways that dramatically affect our lives. The polymerase chain reaction is the almost ubiquitously preferred method for facilitating such genetic analysis; it can be used not only for diagnosing a disease but in telling us what diseases we are more likely to suffer from in later life and how to adjust our lifestyles accordingly. It can even tell us how we will respond to therapeutic drugs such that we can avoid adverse reactions by tailoring dosage or selecting alternative therapies as appropriate. It is in areas such as these that the analytical power and potential of polymerase chain reaction for the provision of genetic analysis has been proven time and time again over the last two decades. However, whilst the success of polymerase chain reaction has been backed by significant financial and technological investment, some would argue that it has also been constrained by a strong patent position such that exploitation has been restricted to a few large players with sufficient financial muscle to enter this market.

\section{THE TECHNICAL CHALLENGE}

Given that a genetic analysis would be desirable, there is the challenge of targeting the relevant piece of sequence information upon which to perform such a test. The human genome is vast, comprising some 3.2 billion base-pairs of information organized into 23 pairs of chromosomes. Within the total genome, there are estimated to be $>30000$ genes often sub-divided and interspersed by noncoding and intervening regions as well as regulatory

Business Development Manager, Life Sciences Division, LGC, Teddington, Middlesex TW11 OLY, UK sequences. Thus, for any specific human disorder, only a minute percentage of a DNA sample is of relevance. Genetic analysis therefore needs to focus on a small region of DNA sequence information and then look for mutations or polmorphisms within it. A technique is consequently required that cannot only generate sufficient DNA of the region of interest but also analyse the genetic sequence within that region. Such is the versatility of polymerase chain reaction.

\section{THE POLYMERASE CHAIN REACTION}

How then to target a specific genetic region for further analysis? There are several routes to accomplish this but, of these, polymerase chain reaction is arguably the most well known and successful. Kary Mullis was awarded the Nobel Prize in Chemistry in 1993 for inventing polymerase chain reaction following his early work at the Cetus Corporation in the 1980s. The idea is said to have been conceived by Mullis in 1983 whilst driving at night through the Californian mountains.

In 1975 Sanger developed an in vitro method for copying DNA sequence in which short oligonucleotides were used to initiate or prime DNA synthesis from a specific site. These 'primers' would be designed to uniquely bind to DNA adjacent to the sequence of interest. Mullis' idea was to use a second oligonucleotide to prime a further round of DNA synthesis returning back along the first product. This process could then be repeated many times using temperature cycling to drive the process of priming, extension and strand separation. In this manner, sequence defined by the position of the primers would be copied many times in a process that has been likened to genetic photocopying.

Whilst simple in concept, the early experiments required fresh DNA polymerase (the enzyme which copied DNA starting from the primer) to be added at each cycle since the strand separation or denaturing step involved heating to $95^{\circ} \mathrm{C}$ in order to enable the priming and synthesis to be repeated. This high temperature caused inactivation of the DNA polymerase. Additionally, the reaction had to be moved by hand between water-baths at appropriate temperatures. However, heat stable DNA polymerases, such as Taq Polymerase from the micro-organism Thermus 


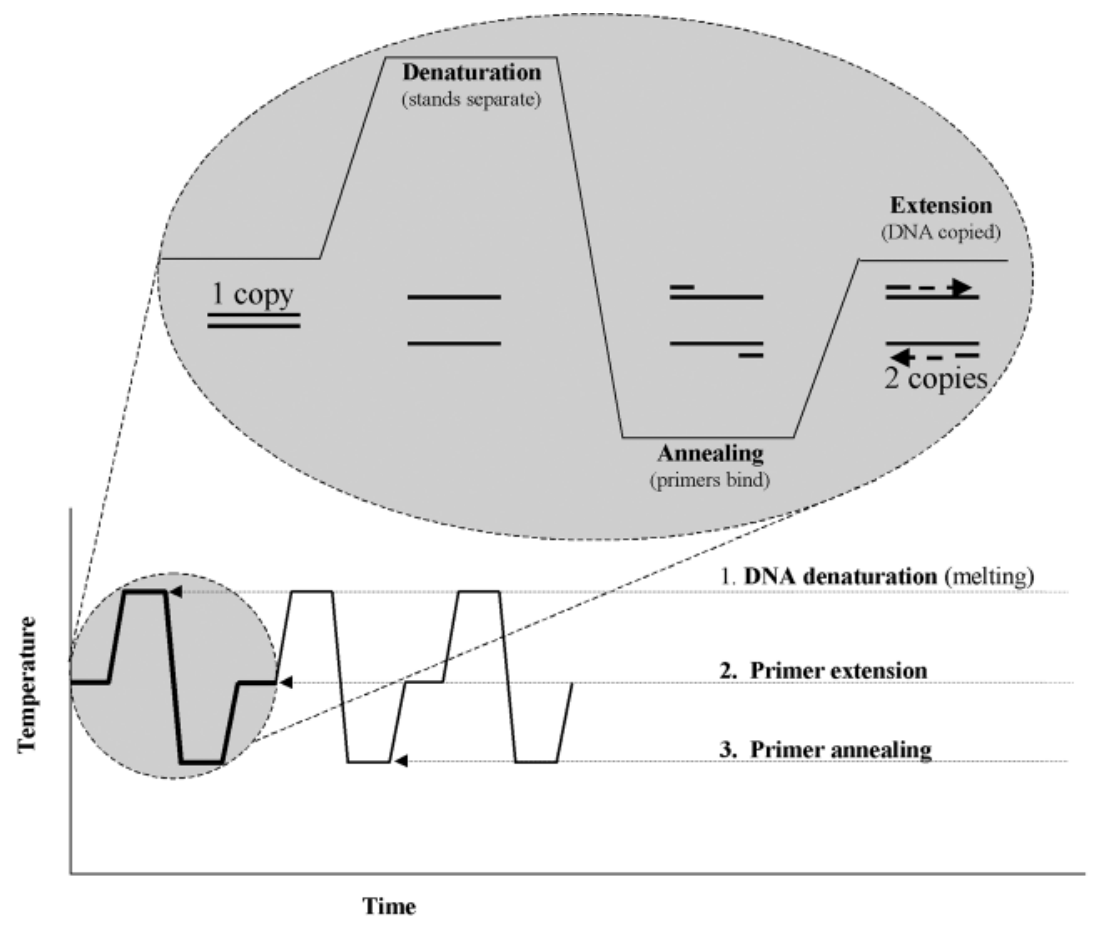

(1) Denaturation: double-stranded DNA molecules are melted or denatured at high temperature $\left(\sim 95^{\circ} \mathrm{C}\right)$ to give single-stranded targets for duplication. (2) Annealing: the temperature is lowered to a suitable annealing temperature $\left(\sim 50^{\circ} \mathrm{C}\right)$. Short synthetic pieces of DNA or primers are bound to the single-stranded target sequences and prime DNA synthesis by Taq polymerase. (3) Extension: the temperature is raised to the optimal temperature for Taq polymerase $\left(\sim 72^{\circ} \mathrm{C}\right)$. A cycle of amplification is completed and the process can be repeated, theoretically doubling the amount of product with each cycle in an exponential manner until a maximal level or plateau is achieved.

Figure 1 The polymerase chain reaction amplification process is driven by repetitive rounds of denaturation, annealing and extension

aquaticus, and genetically engineered forms have since been introduced facilitating the use of automated thermal cyclers, greatly simplifying and improving the process.

There are many variations on the theme of polymerase chain reaction. However, in many cases, the advantage of this genetic amplification technique is that very little DNA is required to initiate the process and that relatively large amounts of specific sequence are generated such that the product may, for many subsequent analytical purposes, be considered pure. It is therefore possible to easily analyse such products by for example sequence length, sequence specific enzymatic digestion, or by a variety of DNA probe systems such as homogeneous or real-time polymerase chain reaction.

\section{THE POST-PATENT OPPORTUNITY}

With the demise of the early process or 'foundational' patents for polymerase chain reaction last year in the USA and many people awaiting their expiry in Europe in March 2006, there has been significant speculation as to the impact this may have on the molecular diagnostics industry which may no longer be restrained by license fees and royalties.

The uses and variants of polymerase chain reaction have grown beyond all expectation since the publication of the first paper by Saiki et al. in 1985 in which the amplification of the Beta Globin gene and its potential use for screening for sickle cell anaemia was described.

Opportunities for molecular diagnostics continue to advance and the market is predicted to grow to $\$ 12$ billion by 2010 and to $\$ 35$ billion by 2015 . With such substantial opportunities it is not surprising that significant investment has been made in technological improvements and developments such as with thermal stable enzymes and real-time polymerase chain reaction and that further patent protection has been sought, effectively extending the lifetime, although perhaps not the dominance, of the polymerase chain reaction process. Whilst further polymerase chain reaction-related patents populate calendars for years to come, the fact that those covering the core process have expired means that techniques exploiting polymerase chain reaction based amplification can now be used without a license. However, whilst accessibility to the basic process is increasing with the demise of these early patents and it is predicted that the cost of enzymes and equipment will fall, real-time polymerase chain reaction methods, such as the $5^{\prime}$ Exonuclease assay, will undoubtedly remain popular and continue to generate significant income for years to come. For example, patents of relevance to thermal stable DNA polymerases will not expire in Europe until 2007 and 2009 
whilst others of relevance to real-time polymerase chain reaction extend to 2010 and beyond.

Consequently, whilst the reduction in license and royalty fees associated with the foundational patents has been heralded as a milestone for those in the field of molecular diagnostics wishing to exploit polymerase chain reaction, the financial impact of associated licenses relating to thermal stable polymerases, real-time polymerase chain reaction and others continues. Additionally, regulatory hurdles associated with the Food and Drug Administration in the USA and In-Vitro Diagnostic Directive within Europe significantly impact on both financial investment and time to market for those within and hoping to enter the molecular diagnostics market. Consequently, it remains to be seen whether there will be an increase in the number of tests based on polymerase chain reaction entering the diagnostics arena or whether regulatory hurdles and other patents relating to the polymerase chain reaction process will continue to restrain developments in this area.

What can we expect from the future? In broad terms, we can envisage our understanding of genetics will continue to develop. Such knowledge, and the desire for exploitation, will continue to drive technological development to enable tests to be performed in an ever-simpler, faster and cost effective manner. In the shorter term, we can expect the analysis of some of the simple gene disorders to become accessible in a point-of-care setting. Similarly, we can expect the testing of more complex gene disorders to be increasingly undertaken within specialist analytical centres. Polymerase chain reaction will undoubtedly continue to play a highly significant role in the provision of these services. However, we may also expect increasing competition from other technologies, exploiting the now expired core process and seeking to minimise or avoid entirely the financial impact of other, still valid patents. Increasing competition within the market will undoubtedly drive testing capability, reduce analytical costs and increase competition from private sector suppliers. How such costs will be met is as yet uncertain. However, the demands of the ever better informed patient with access to the internet will doubtless continue to increase pressure for such health care services once concerns over any potentially negative impact on employment and medical insurance have been allayed. The polymerase chain reaction will doubtless be with us for many years to come.

Competing interests David McDowell is an employee of LGC, a leading provider of analytical services including those exploiting polymerase chain reaction.

\section{REFERENCES}

1 Saiki RK, Scharf S, Faloona F, et al. Enzymatic amplification of betaglobin genomic sequences and restriction site analysis for diagnosis of sickle cell anemia. Science 1985;230:1350-4

2 Sanger F, Coulson AR. A rapid method for determining sequences in DNA by primed synthesis with DNA polymerase. $J$ Molec Biol 1975;94:441-8 